\title{
Movement Modeling and Control for Robotic Bonnet Polishing
}

\section{Zewen LIN}

Xiamen University

Zhenzhong Wang ( $\nabla$ wangzhenzhong@xmu.edu.cn )

https://orcid.org/0000-0003-3917-1619

Xuepeng HUANG

Xiamen University

\section{Original Article}

Keywords: Robotic Polishing, Bonnet polishing, Precession movement, Control

Posted Date: November 17th, 2020

DOI: https://doi.org/10.21203/rs.3.rs-106436/v1

License: (c) (1) This work is licensed under a Creative Commons Attribution 4.0 International License. Read Full License 


\section{Title page}

\section{Movement Modeling and Control for Robotic Bonnet Polishing}

Ze-Wen Lin, born in 1995, is currently a master at Shenzhen Research Institute of Xiamen University, China.

E-mail: zevin_lin@163.com

Zhen-Zhong Wang, born in 1981, is currently an associate professor at Shenzhen Research Institute of Xiamen University, China. He received his $\mathrm{PhD}$ degree from Xiamen University, China, His research interests include intelligent manufacturing and precision engineering.

E-mail: wangzhenzhong@xmu.edu.cn

Xue-Peng-Huang, born in 1995, is currently a master at Shenzhen Research Institute of Xiamen University, China.

E-mail: 1146524731@qq.com

Corresponding author: Zhen-Zhong Wang E-mail: wangzhenzhong@xmu.edu.cn 


\title{
Movement Modeling and Control for Robotic Bonnet Polishing
}

\author{
Zewen Lin $^{1,2}$, Zhenzhong Wang ${ }^{1,2} *$ and Xuepeng Huang ${ }^{1,2}$
}

\begin{abstract}
The spin axis of bonnet tool maintains a constant angle (precession angle) with the normal of polishing point in polishing. In this paper, a controlled model was established on robotic machining platform to polish large diameter axisymmetric aspheric optics. Based on the transformation relationship in spatial coordinate system, the relationships between workpiece coordinate system, polishing point local coordinate system and the bonnet tool coordinate system were set up respectively. So that the movement model of bonnet precession polishing was obtained. What's more, the efficiency optimal control was added to the movement model. the trajectories and poses of the polishing were determined, and the change of robot linkage angle difference was obtained. Finally, the precession movement model and the control algorithm were verified by the simulation in the Robotstudio, an offline simulation software and experiment for plane component.
\end{abstract}

Key words: Robotic Polishing, Bonnet polishing, Precession movement, Control

\section{Introduction}

Bonnet polishing technology has successfully applied to the polishing of optical components, which firstly proposed by the British ZEEKO company and University of London in the early 21st century [1]. It uses an inflatable-sphericalflexible rubber as polishing tool, and adopts a special precession polishing method, that the rotation axis of bonnet and local normal are maintained at a certain angle (precession Angle) during the polishing process. As an emerging polishing technology, bonnet polishing technology has the advantages of high polishing efficiency and processing precision, besides it has great advantages in polishing free-form optical lenses. In China, Harbin Institute of Technology [2], Xiamen University [3] and so on have carried out research in the field of bonnet polishing.

Industrial robots do well in reducing development cost, working efficiently, and machining adaptability [4], which have made more and more researchers value robot polishing at home and abroad. Fusaomi Nagata ${ }^{[5]}$ et al, JA Dieste ${ }^{[6]}$ and Professor Ji ShiMing ${ }^{[7]}$ of Zhejiang University of Technology have made progress in robotic polishing. Movement modeling and control is the basis for practical polishing.

Pan ${ }^{[8]}$ established precession-movement model and control algorithm for the five-axis bonnet machine, which polishing large axisymmetric aspheric optical component polishing. Different from the bonnet machine's control mode, which position and orientation is controlled by the XYZ linear axis and the AB rotating axis. Industrial robot, a six-joints structure, controls the joint turning angles to ensure tool center point (TCP) reach predetermined position and tool coordinate system satisfies the precession posture. In this paper, ABB robot equipment and special bonnet polishing tool are taken as research objects, the robot precession movement model is established and the optimal efficiency control algorithm is proposed.

\section{Modeling for robotic precession movement}

The key of robotic precession model, that robotic end-effector maintaining the precession attitude at any polishing point, is to control the bonnet tool coordinate system to maintain the corresponding posture. Different brands of robots adopt different control methods in position and pose control of end-effector, and ABB robots adopt position values and quaternions. The robotic bonnet polishing system can achieve precession polishing in two aspects: 1 . Determine the position of polishing point. Determine polishing path and discretization, and obtain the coordinates of bonnet center (TCP) through coordinate transformation; 2. Determine the precession pose, Coordinate relations are obtained through coordinate transformation, so that transformation matrix is determined and the quaternions of pose are solved.

Fig. 1 shows the precession model of the robotic bonnet polishing. Workpiece coordinate system

$O_{w} x_{w} y_{w} Z_{w}$, tool coordinate system $O_{t} x_{t} y_{t} z_{t}$ and local coordinate system $O_{p} x_{p} y_{p} z_{p}$ are established respectively at workpiece center, bonnet tool center and arbitrary polishing point. The workpiece coordinate system direction is consistent with robotic base coordinate system $O_{0} x_{0} y_{0} Z_{0}$, and the tool coordinate system is in the same direction as the sixth joint coordinate system .In the local coordinate system, $z_{p}$ is opposite to the normal vector of polishing point, $y_{p}$ is

Corresponding author E-mail: wangzhenzhong@xmu.edu.cn, 
the tangent vector on the ZY section, and $x_{p}$ direction is uniquely determined by the right-handed principle.$\rho$ is the precession angle, $\varepsilon$ is the angle between the spin axis of bonnet in the $x_{t} y_{t}$ plane and $x_{t}$ direction. From the definition of the precession angle, the $\varepsilon$ angle is between $0-360\left[^{\circ}\right]$, and $\mathrm{r}$ represents bonnet radius.

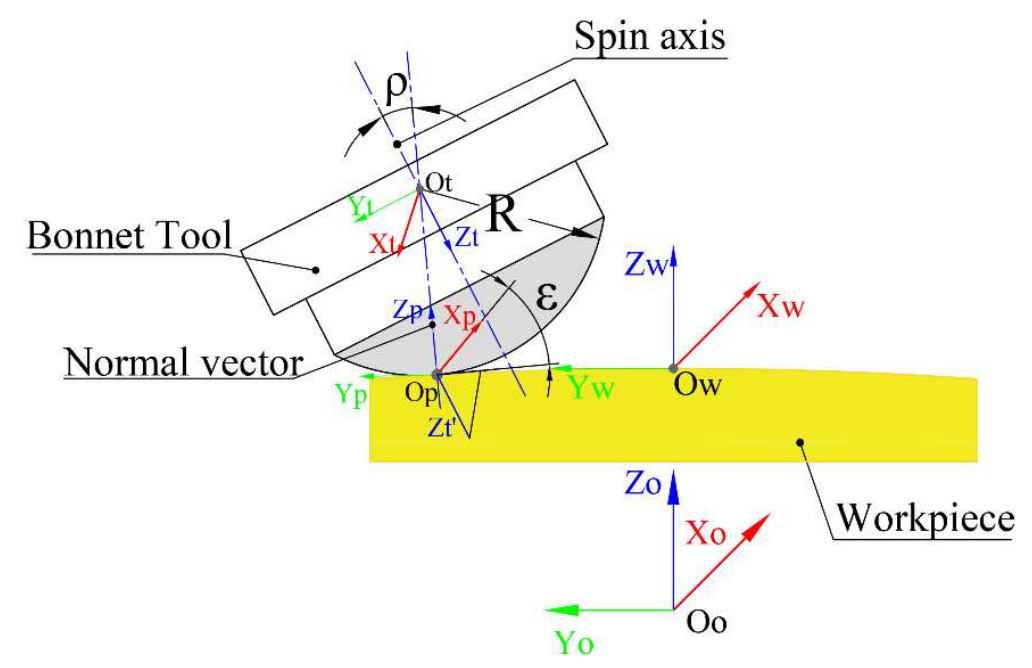

Fig. 1 Precession model of the robotic bonnet polishing

The surface function of large axisymmetric aspheric optical component can be expressed as Eq.1.

$F\left(\begin{array}{lll}x & y & z\end{array}\right)=0$

Eq.2 is normal vector of polishing point $\left(\begin{array}{llll}x_{p} & y_{p} & z_{p}\end{array}\right)$.

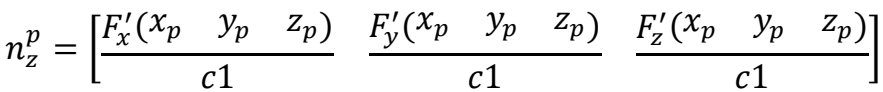

Tangent vector on $\mathrm{ZY}$ section of polishing point can be given as Eq.3.

$n_{y}^{p}=\left[\begin{array}{lll}0 & \frac{y_{p}}{c 2} & \frac{-y_{p} F_{x}^{\prime} / F_{z}^{\prime}}{c 2}\end{array}\right]$

Combined with Eq.2 and Eq.3, the vector of X direction can be expressed as Eq.4:

$n_{x}^{p}=\left[\begin{array}{lll}\frac{-\left(F_{z}^{\prime} F_{z}^{\prime}+F_{x}^{\prime} F_{y}^{\prime}\right) / F_{z}^{\prime}}{c 1 c 2} & \frac{F_{x}^{\prime} F_{x}^{\prime} / F_{z}^{\prime}}{c 1 c 2} & \frac{F_{x}^{\prime}}{c 1 c 2}\end{array}\right]$

Where $c 1=\sqrt{\left(F_{x}^{\prime}\right)^{2}+\left(F_{y}^{\prime}\right)^{2}+\left(F_{z}^{\prime}\right)^{2}}, \mathrm{c} 2=\sqrt{y_{0}^{2}+\left(-y_{0} F_{x}^{\prime} / F_{z}^{\prime}\right)^{2}}$.

So ${ }^{w} R_{p}$ that the transformation matrix of local coordinate system $O_{p} x_{p} y_{p} z_{p}$ to workpiece coordinate system $O_{w} x_{w} y_{w} z_{w}$ can be obtained.

Since the angle $\varepsilon$ can vary within the range of $0-360\left[^{\circ}\right]$, The spin axis of bonnet precession polishing are distributed on the conical plane as Fig. 2 shown, that schematic diagram of spin axis distribution of bonnet. 


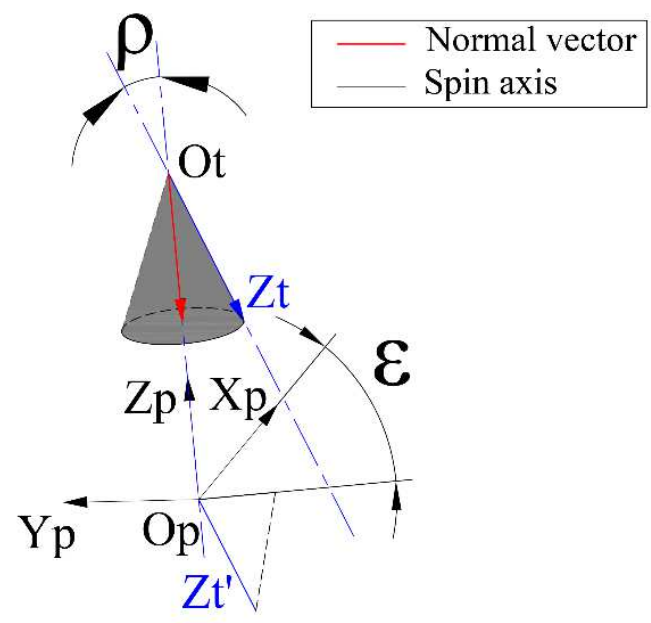

Fig. 2 Schematic of spin axis distribution of bonnet

Base on coordinate transformation, the transformation relation, the local coordinate system $O_{p} x_{p} y_{p} z_{p}$ to the tool coordinate system $O_{t} x_{t} y_{t} z_{t}$ can be shown as Eq.5.

$$
{ }^{p} \mathrm{R}_{t}=\operatorname{Rot}(Z, \varepsilon) \operatorname{Rot}(Y, \rho) \operatorname{Rot}(Z,-\varepsilon)
$$

So position of bonnet center can be expressed as Eq6.

$$
\begin{aligned}
& x_{t}=x_{p}+\left(F_{x}^{\prime} \times r\right) / c_{1} ; \\
& y_{t}=y_{p}+\left(F_{y}^{\prime} \times r\right) / c_{1} ; \\
& z_{t}=z_{p}+\left(F_{z}^{\prime} \times r\right) / c_{1}
\end{aligned}
$$

The pose transformation matrix as Eq.7 can be obtained.

$$
{ }^{w} R_{t}={ }^{w} R_{p}{ }^{p} R_{t}=\left(n_{x} ; n_{y} ; n_{z}\right)
$$

In the actual motion programming of robot, the transformation matrix needs to be converted into quaternions $\left[\begin{array}{llll}\text { q1 } & \text { q2 } & \text { q3 } & \text { q4}\end{array}\right]$ according to certain rules, shown as Eq.8.

$$
\begin{aligned}
& q_{1}=\frac{\sqrt{n x_{1}+n y_{2}+n z_{3}+1}}{2}, \\
& q_{2}=\frac{\sqrt{n x_{1}-n y_{2}-n z_{3}+1}}{2}, \operatorname{sign}\left(q_{2}\right)=\operatorname{sign}\left(n y_{3}-n z_{2}\right), \\
& q_{3}=\frac{\sqrt{n y_{2}-n x_{1}-n z_{3}+1}}{2} \operatorname{sign}\left(q_{3}\right)=\operatorname{sign}\left(n z_{1}-n x_{3}\right), \\
& q_{4}=\frac{\sqrt{n z_{3}-n x_{1}-n y_{2}+1}}{2}, \operatorname{sign}\left(q_{4}\right)=\operatorname{sign}\left(n x_{2}-n y_{1}\right) .
\end{aligned}
$$

The value of quaternions are determined by the surface function $F\left(\begin{array}{lll}x & y\end{array}\right)$, precision-angle $\rho$ and angle $\varepsilon$. Endeffector's position and pose quaternions can be obtained to control the robot motion by establishing the movement model. Since the value of angle $\varepsilon$, that change between $0^{\circ}$ and $360^{\circ}$, can be determined by a feasible control algorithm, then the pose of the bonnet tool can be determined.

\section{Control for robotic bonnet polishing movement}

Reducing processing cycle and improving processing efficiency in industrial production are beneficial to increase profit. Optimizing feeding time is an effective and feasible method to improve polishing efficiency. Robot with 6 rotary joints adopt a six axis simultaneous motion mode. In the case of a given feeding rate, feeding time depends on a time required for 
maximum joint from point to point. Therefore, the maximum change in angle of robotic joint rotation at any two points is taken as the optimization target, it can be shown as Eq.9.

$$
\Delta \theta=\max \left[\left|\Delta \theta_{1}\right| \quad\left|\Delta \theta_{2}\right| \quad\left|\Delta \theta_{3}\right| \quad\left|\Delta \theta_{4}\right| \quad\left|\Delta \theta_{5}\right| \quad\left|\Delta \theta_{6}\right|\right]
$$

A optimal efficiency control for robotic bonnet polishing is proposed.To make the change of angle minimum between any two polishing points during polishing movement, bonnet poses are optimized. The rotating angles of robotic six joints are solved by combining the forward and inverse kinematics of the robot with MATLAB by using the transformation matrix of the coordinate system. The flow of control algorithm is shown in Fig.3.

Define coordinate system of workpiece and input parameters include
surface function, workpiece length $(L)$ and width $(W)$, grating step
length $\Delta x, \Delta y$, precession angle $\rho$, bonnet radius $R$, initial angle $\varepsilon$,
robot's initial parameter.

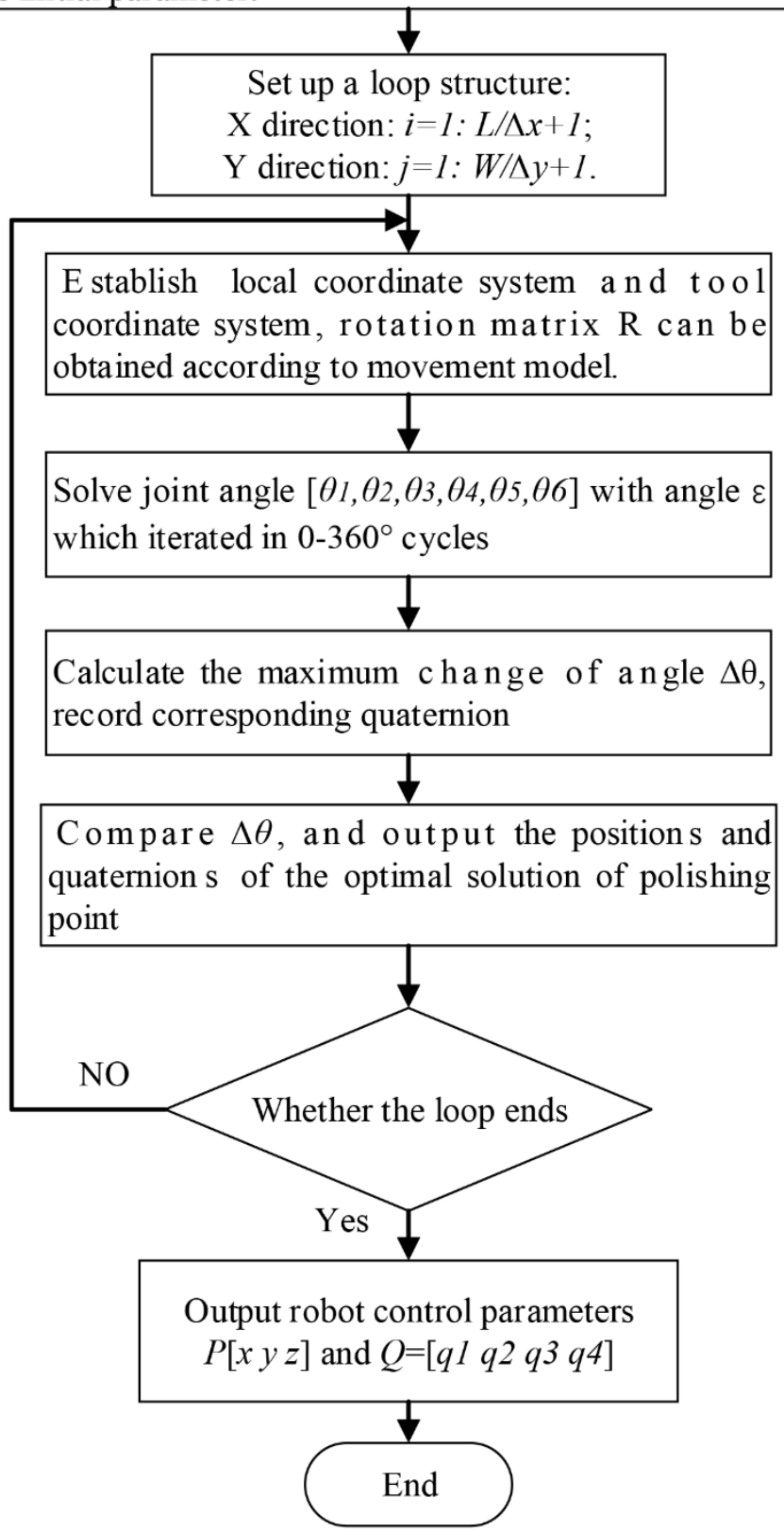

Fig.3 The flow of control algorithm 
The surface function of aspheric optical workpiece is shown as Eq.10.

$$
z=\frac{-C x^{2}}{1+\sqrt{1-(1+K) C^{2} x^{2}}}
$$

The size of polishing area is $400 \times 400$ [mm], precession angle $\rho$ is $20\left[^{\circ}\right]$. XY-linear-grating polishing path is adopted, path spacing and polishing step length $20[\mathrm{~mm}]$. The movement model and control strategy established are used for simulation. The position distribution of bonnet center (TCP) during bonnet polishing process is shown in Fig.4. Angle-change value and program parameters of robot are obtained by the pose control. The curve of maximum change for robotic joint angle from the initial state of bonnet polishing to the first grating scanning path is shown in Fig.5. 


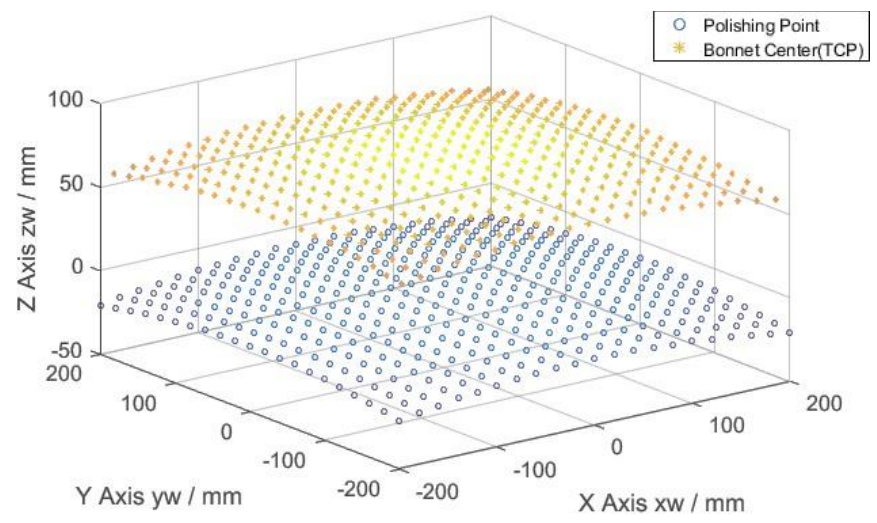

Fig.4 Position distribution of bonnet center (TCP)

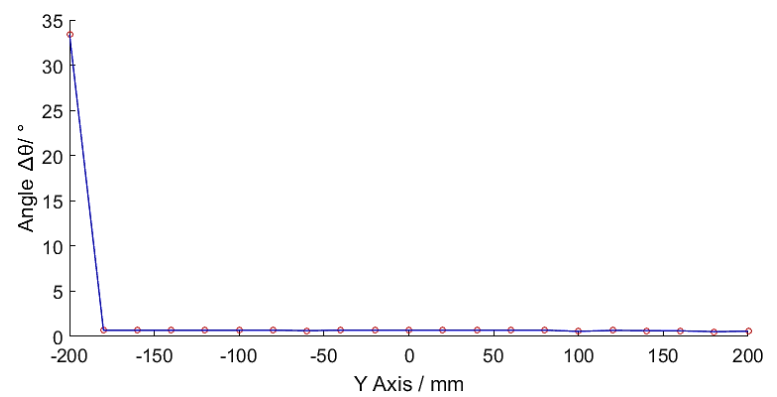

Fig.5 Maximum change of robotic joint angle during bonnet polishing process

The distribution of bonnet center is consistent with that of aspheric surface, which can verify the accuracy of its position control. In pose control, a large angular travel is required to move from the initial position to the first polishing point, while the aspheric curvature changes a little between any two polishing points, so that robot's joint angles change a little. A control code for robot generated by the program is imported into Robotstudio, a special simulation software for ABB robots, in order to ensure the accuracy and effectiveness of the control program. Fig. 6 shows the offline simulation of bonnet polishing in Robotstudio. Simulation shows that the control method is accurate and effective. simulation time is $126.9 \mathrm{~s}$ in total in case of speeding rate is $100 \mathrm{~mm} / \mathrm{s}$ and regardless of polishing dwell time.

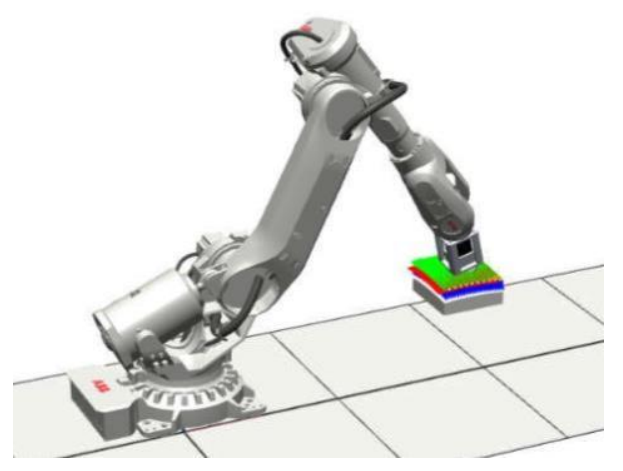

Fig.6 Off-line simulation of bonnet polishing in Robotstudio

This control algorithm is used to polish the plane optical element with the system of robotic bonnet polishing that as Fig.7 shown. Polishing area is $40 \mathrm{~mm} \times 40 \mathrm{~mm}$, velocity of TCP is $0.5 \mathrm{~mm} / \mathrm{s}$, another polishing parameters are shown in table 1 . 


\begin{tabular}{|c|c|c|c|c|}
\hline Bonnet's radius $(\mathrm{mm})$ & $\begin{array}{l}\text { Polishing area } \\
(\mathrm{mm})\end{array}$ & $\begin{array}{c}\text { Precession angle } \\
\left(^{\circ}\right)\end{array}$ & $\begin{array}{l}\text { Z-offset } \\
(\mathrm{mm})\end{array}$ & $\begin{array}{l}\text { Inflation pressure } \\
\qquad(M p a)\end{array}$ \\
\hline 80 & $40 \times 40$ & 20 & 0.4 & 0.2 \\
\hline
\end{tabular}

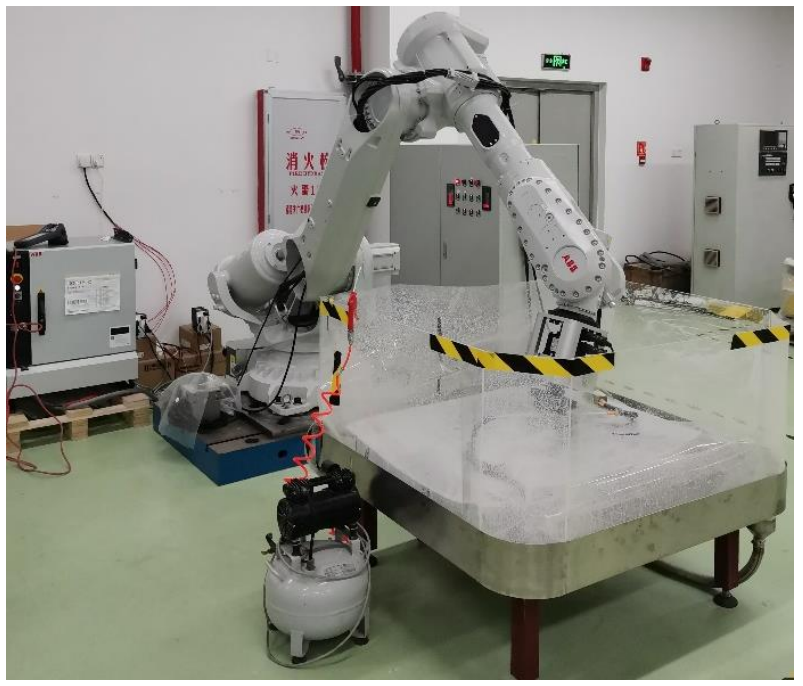

Fig.7 the system of robotic bonnet polishing

There is an obvious polishing area after polishing, as is shown in Fig 8. Taylor Hobson Form Talysurf was used to detect the polished area, the results are shown in Fig 9. Where (a) is surface profile along the red line which shown in Fig 8, and the removal depth was measured at $18 \mu \mathrm{m}$; (b) is the surface profile measured in part of red line, with a PV value of $0.4 \mu \mathrm{m}$. Finally, roughness at $\mathrm{ABCD}$ point were measured, with an average value of Ra $1.4 \mathrm{~nm}$. Experiments can verify the accuracy of the control algorithm and the high efficiency of bonnet polishing.

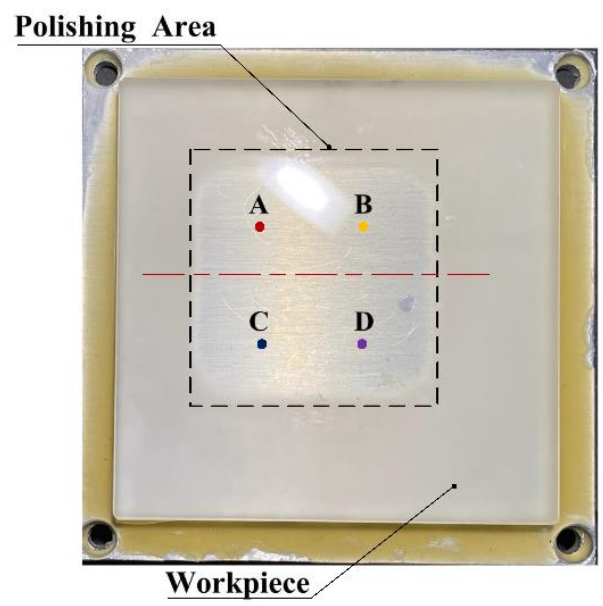

Fig.8 Optical component after polishing
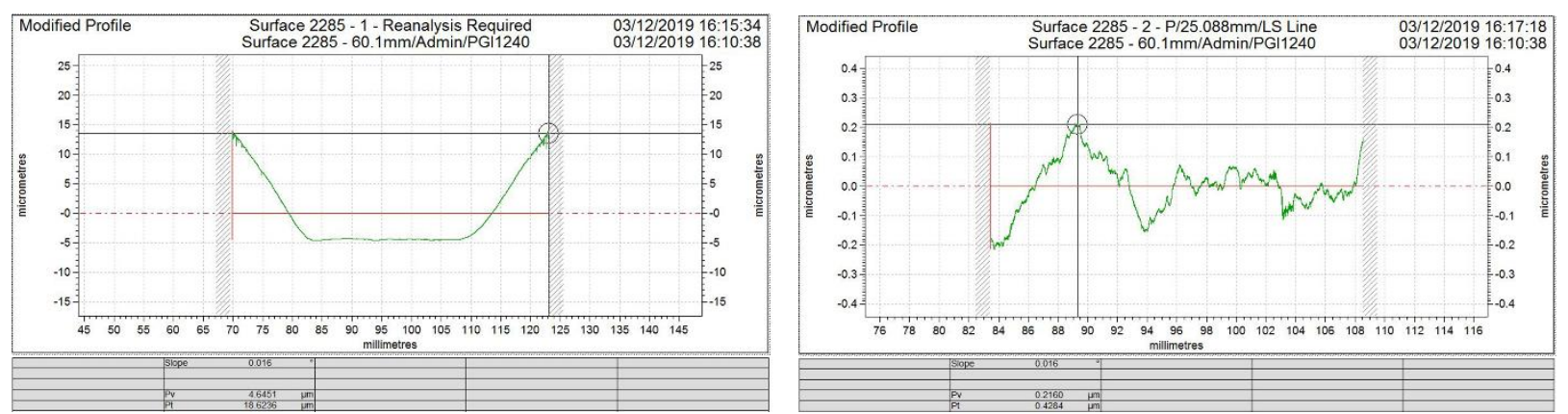


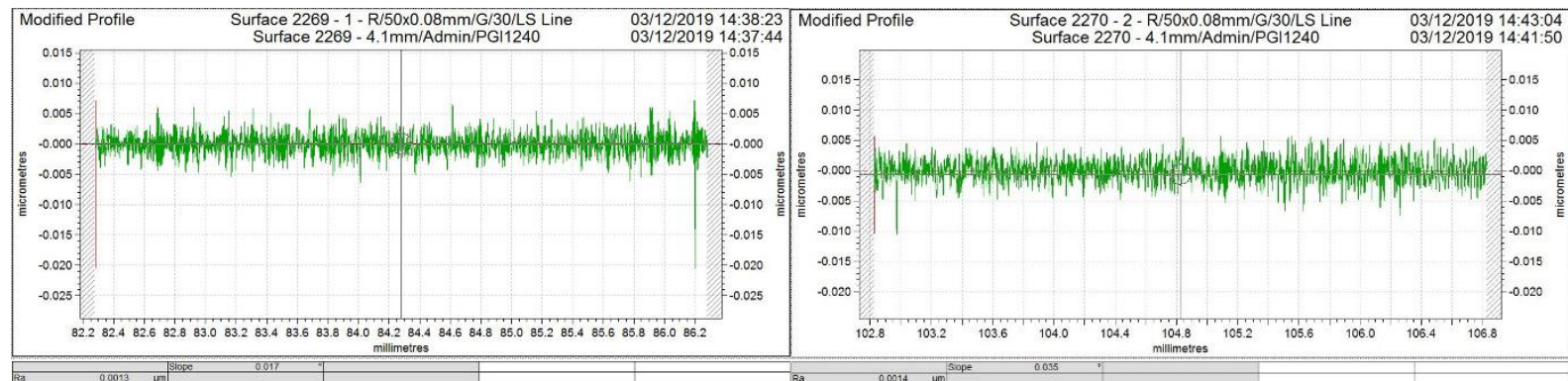

(1)

(2)

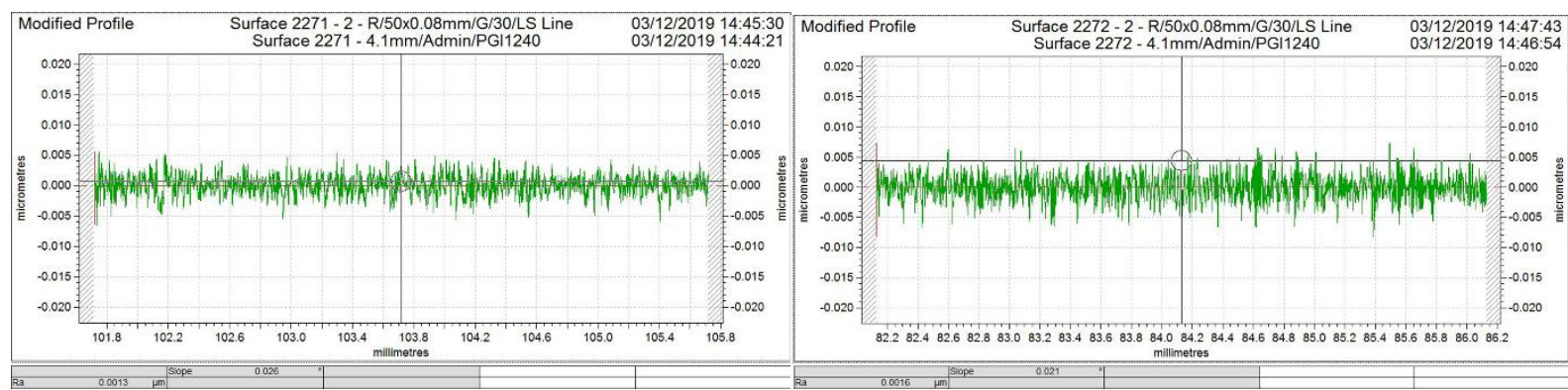

(3)

(4)

(c) Result of roughness at $\mathrm{ABCD}$ point

Fig.9 Measurement results by Taylor Hobson Form Talysurf

\section{Conclusion}

The characteristics of bonnet polishing process were analyzed, and the corresponding model of robot bonnet polishing precession model was proposed. By using robot toolbox in Matlab, inverse solutions of robot motion angles were obtained. Based on the special simulation software for ABB robots, the accuracy of precession control algorithm of the robot bonnet polishing for aspheric component was verified. Finally, algorithm has been successfully applied to polishing with a good result.

\section{Declaration}

\section{Acknowledgements}

The authors sincerely thanks to Professor Wang of Xiamen University for his critical discussion and reading during manuscript preparation.

\section{Funding}

Supported by Science and technology projects of Shenzhen (JCYJ20180306172924636).

\section{Availability of data and materials}

The datasets supporting the conclusions of this article are included within the article. 


\section{Authors' contributions}

The author' contributions are as follows: Zhenzhong Wang was in charge of the whole trial; Zewen Lin wrote the manuscript; Xuepeng Huang assisted with sampling and laboratory analyses.

\section{Competing interests}

The authors declare no competing financial interests.

\section{Consent for publication}

Not applicable

\section{Ethics approval and consent to participate}

Not applicable 


\section{References}

[1] Walker D, Brooks D, King A, et al. The "Precessions" tooling for polishing and figuring flat, spherical and aspheric surfaces[J]. Optics Express, 2003, 11(8):958-964.

[2] Zhang Wei, Li Hongyu, Yu Guoyu. Current Situation of Ultra-Precision Bonnet Polishing Key Technology of Optical Elements[J]. Chinese Acta Optica Sinica, 2009, 29(01): 000027-34.

[3] Ri P, Zhen-Zhong W, Chun-Jin W, et al. Research on control optimization for bonnet polishing system[J]. International Journal of Precision Engineering and Manufacturing, 2014, 15(3):483-488.

[4] Ji Shiming, Huang Xihuan. Review of development and application of industrial robot technology[J]. Chinese Mechanical \& Electrical Engineering Magazine, 2015, 32(1):1-13.

[5] Nagata F, Hase T, Haga Z, et al. CAD/CAM-based position/force controller for a mold polishing robot[J]. Mechatronics, 2007, 17(4-5):207-216.

[6] Dieste J A, Fernández, A, Roba D , et al. Automatic Grinding and Polishing Using Spherical Robot[J]. Procedia Engineering, 2013, 63:938-946.

[7] Ji Shiming, Jin Mingsheng, Zhang Xian, et al. Novel gasbag polishing technique for free-from mold[J]. Chinese Journal of Mechanical Engineering, 2007,43(8): 2-6.

[8]Pan Ri, Wang Zhenzhong, Guo Yinbiao, et al. Movement Modeling and Control of Precession Mechanism for Bonnet Tool Polishing Large Aixsymmetrical Aspheric Lenses [J]. Chinese Journal of Mechanical Engineering , 2012, 48(11):183-190 
Figures

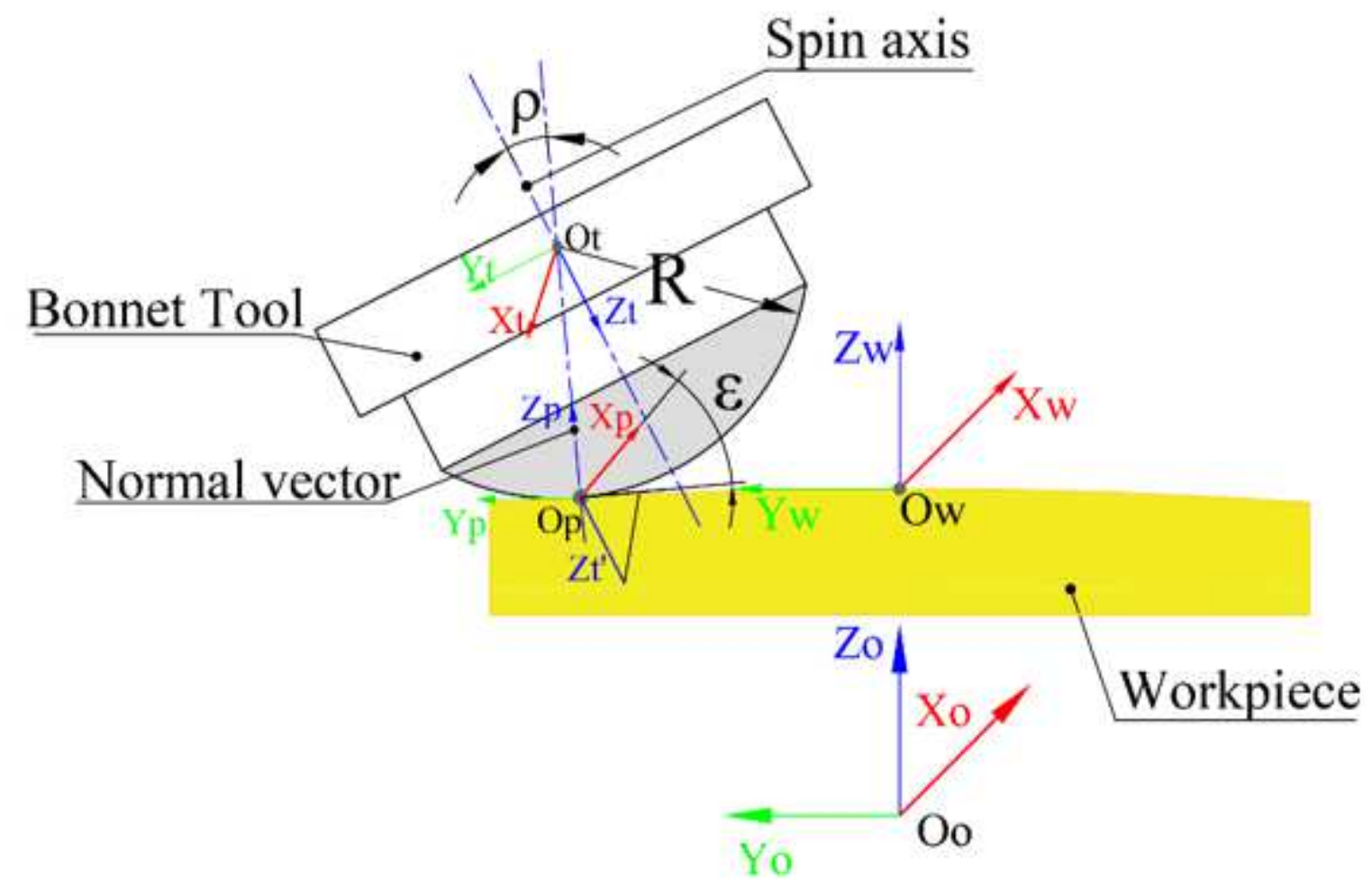

Figure 1

Precession model of the robotic bonnet polishing 


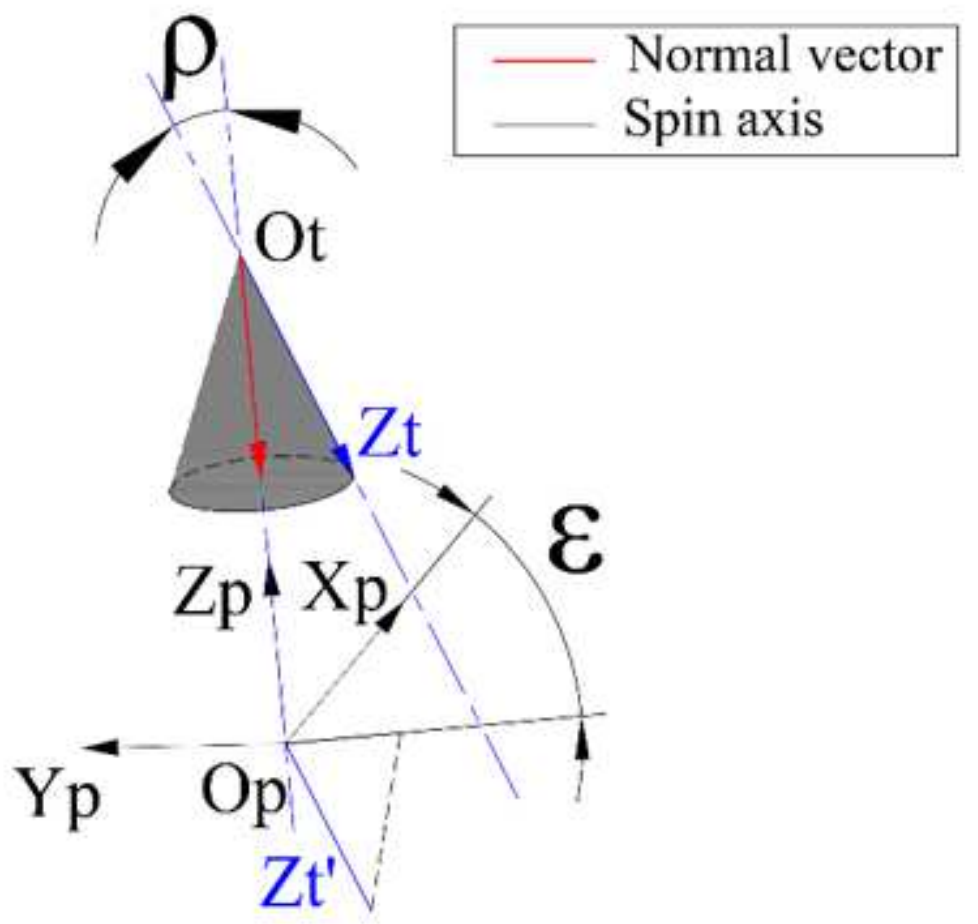

Figure 2

Schematic of spin axis distribution of bonnet 
Define coordinate system of workpiece and input parameters include surface function, workpiece length $(L)$ and width $(W)$, grating step length $\Delta x, \Delta y$, precession angle $\rho$, bonnet radius $R$, initial angle $\varepsilon$, robot's initial parameter.

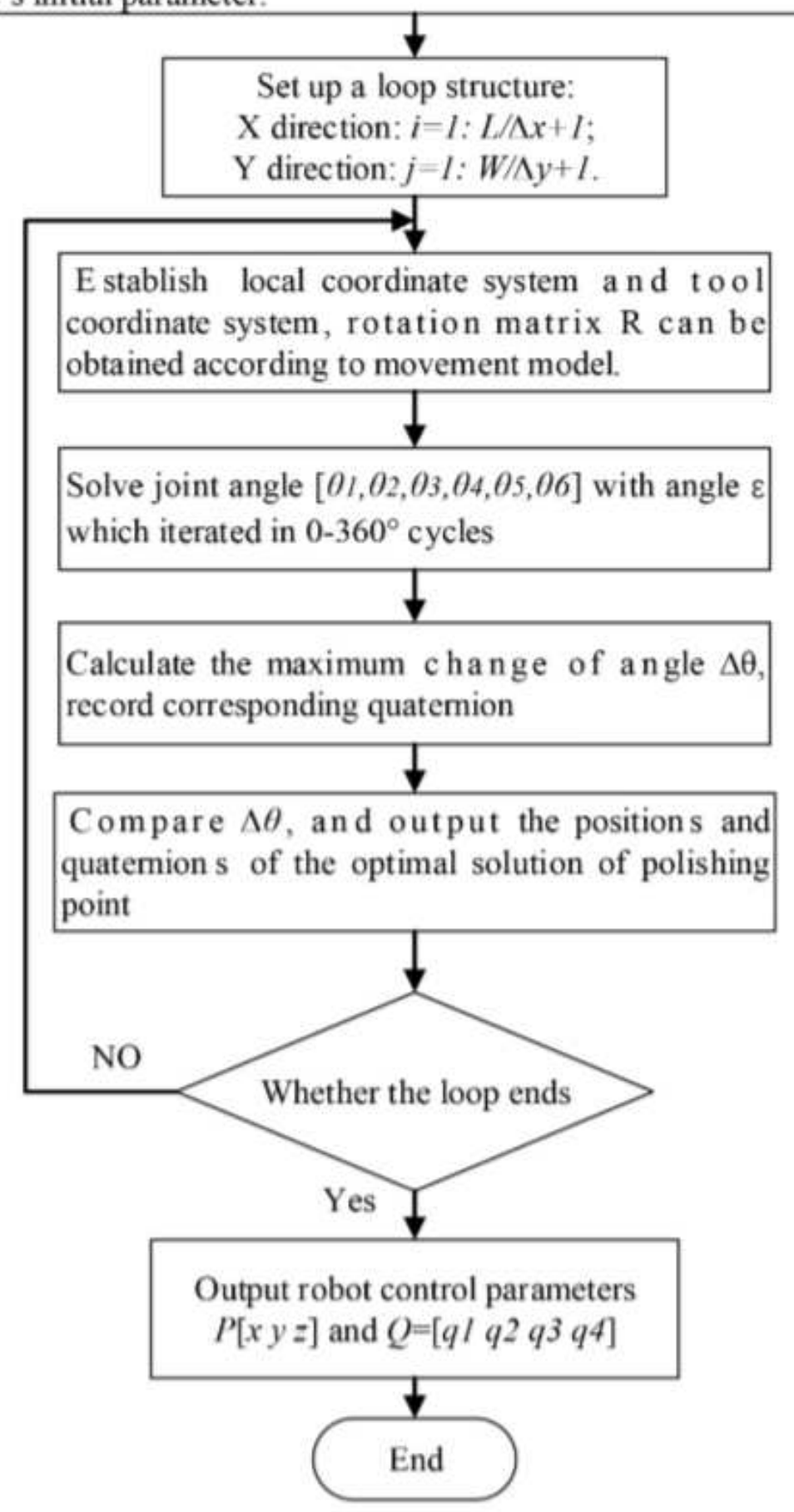

\section{Figure 3}

The flow of control algorithm 


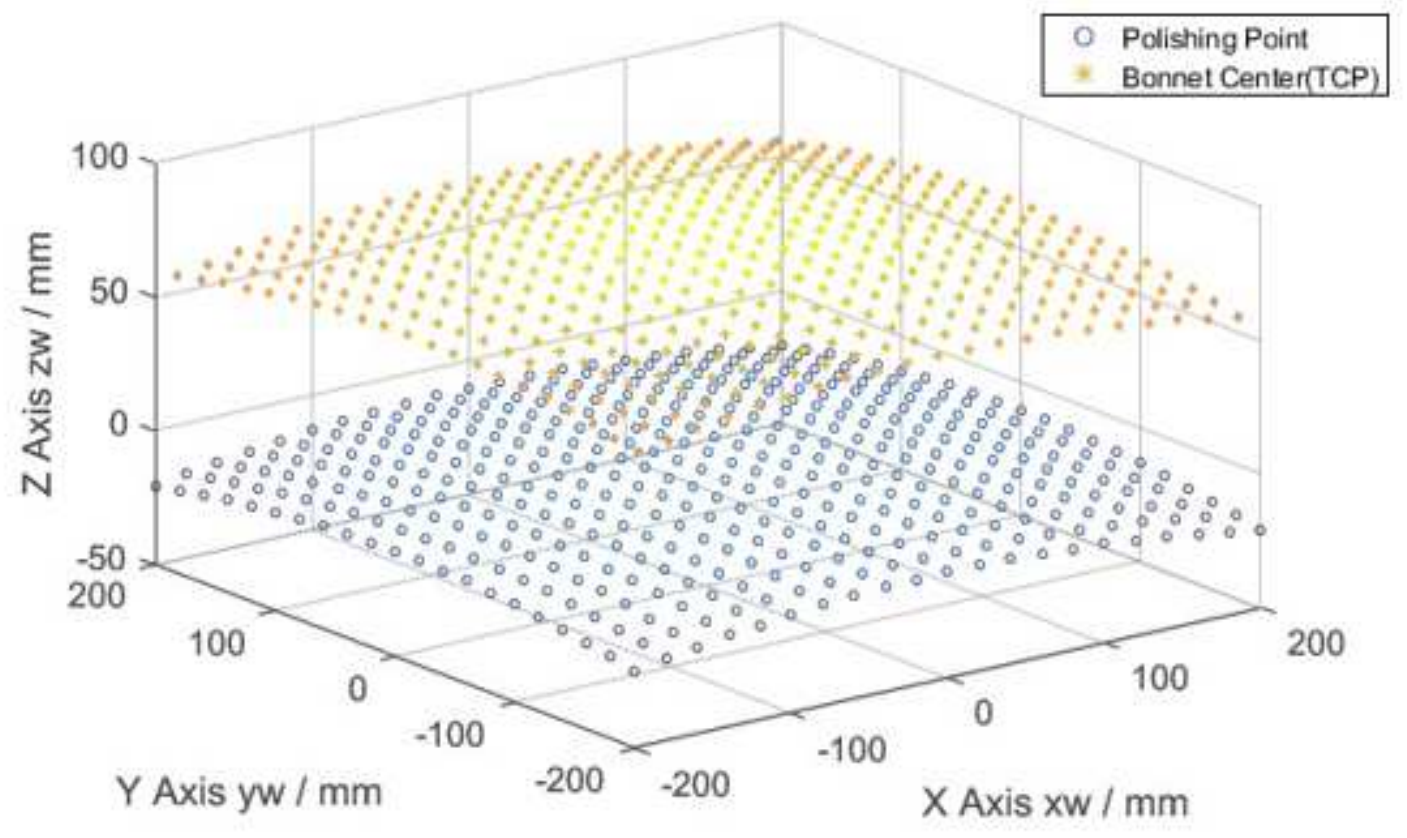

Figure 4

Position distribution of bonnet center (TCP)

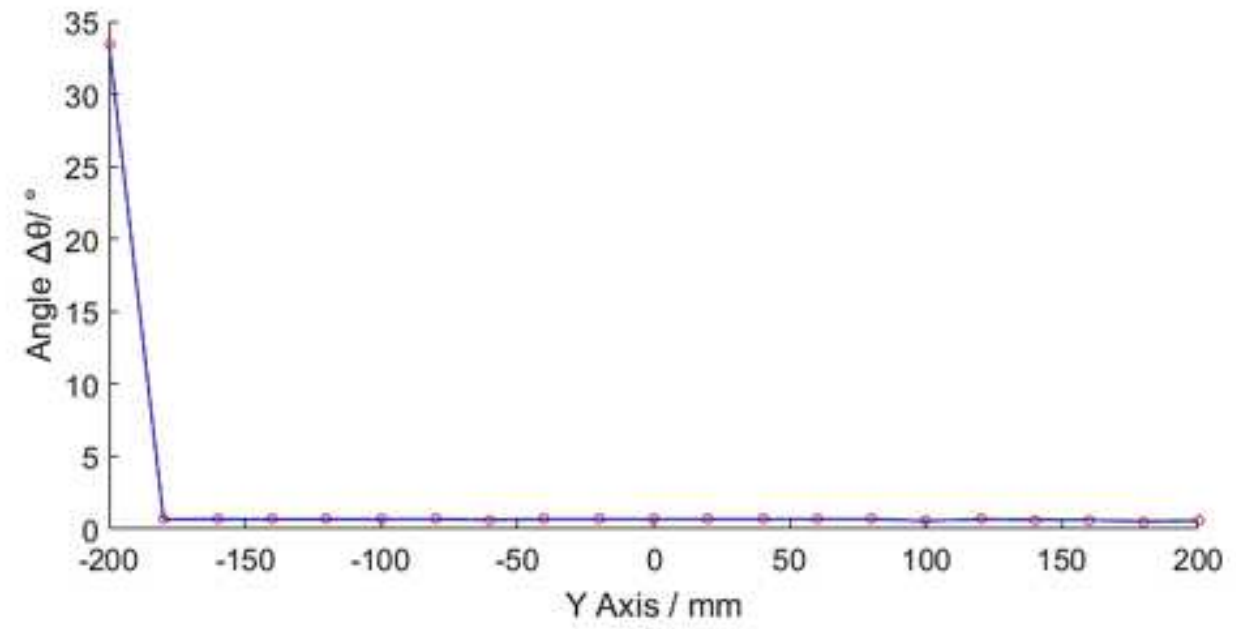

\section{Figure 5}

Maximum change of robotic joint angle during bonnet polishing process 


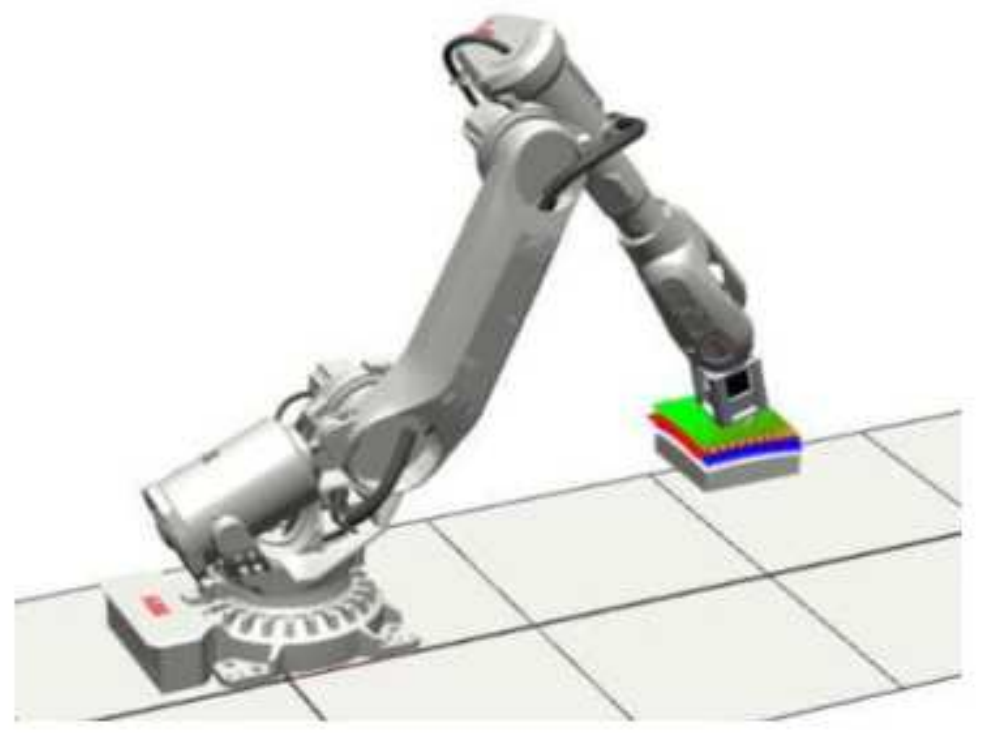

Figure 6

Off-line simulation of bonnet polishing in Robotstudio

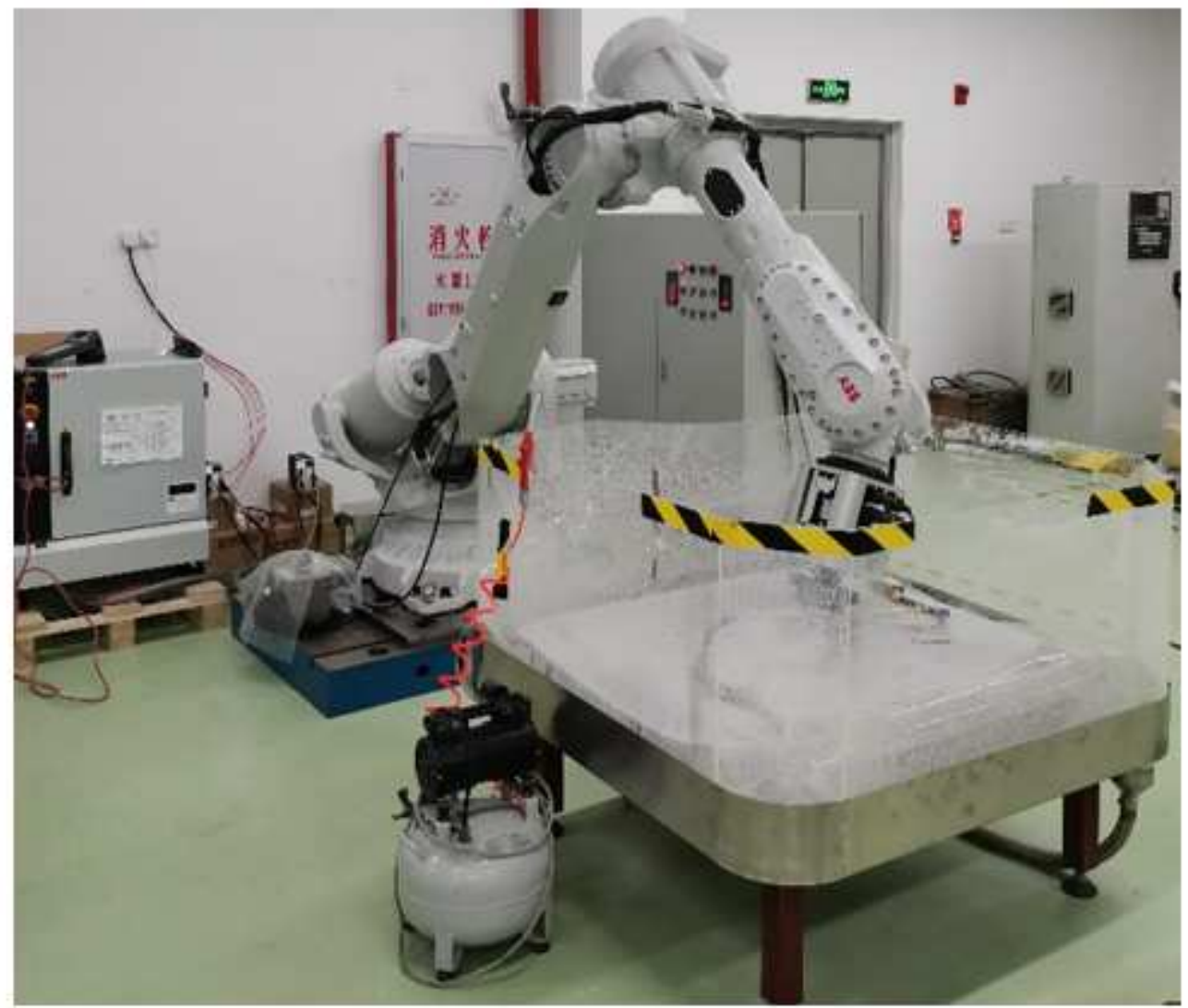

Figure 7

the system of robotic bonnet polishing 


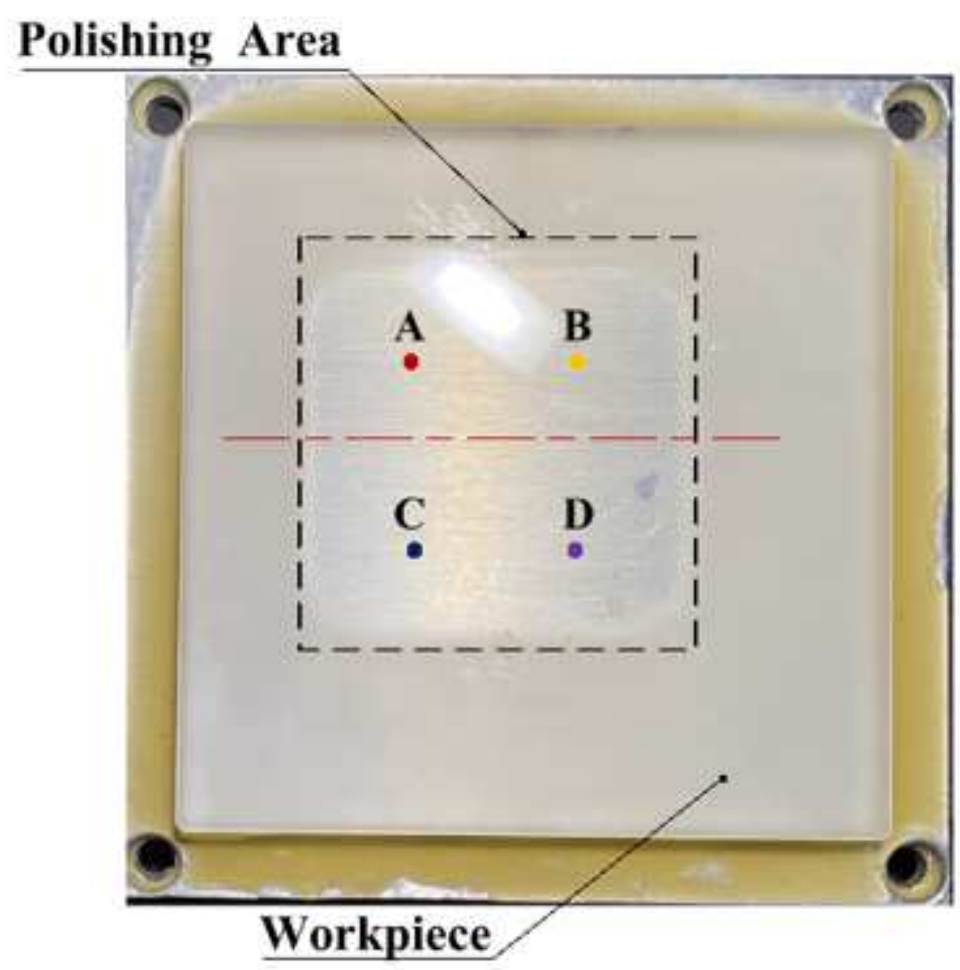

Figure 8

Optical component after polishing 


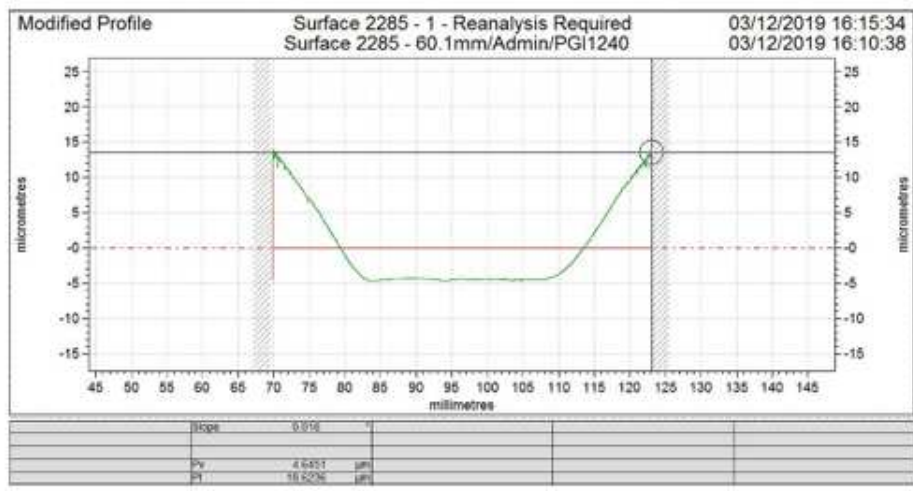

(a) Surface profile along the red line

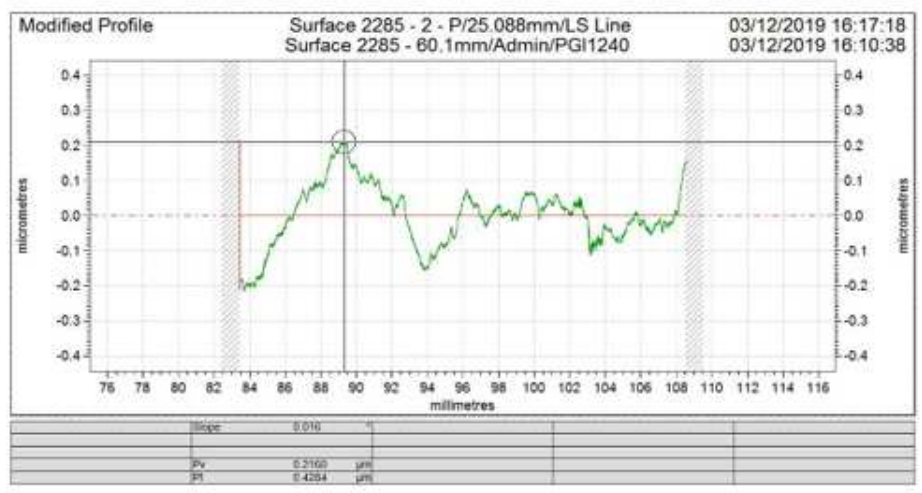

(b) Surface profile along part of red line

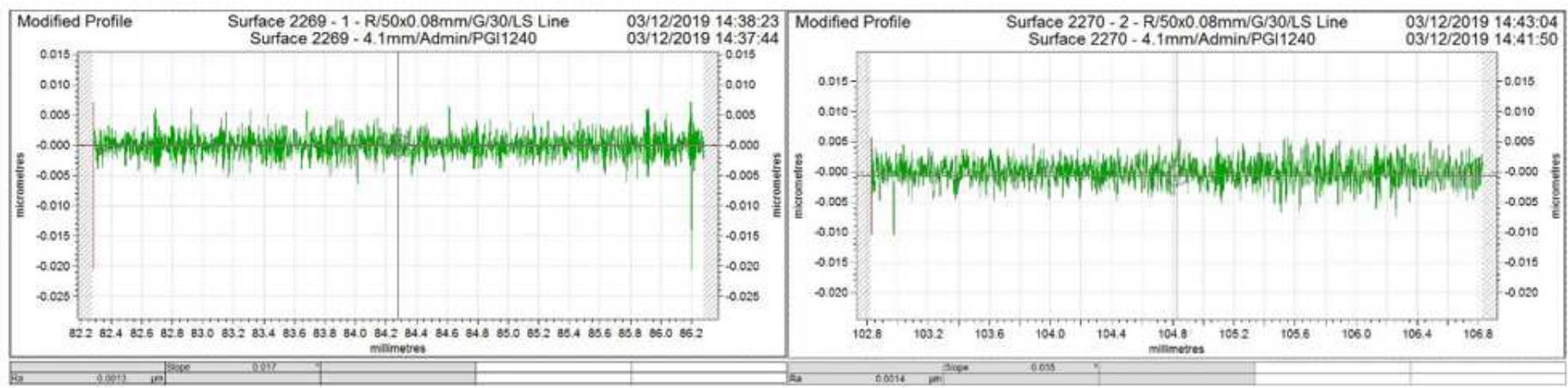

(1)

(2)

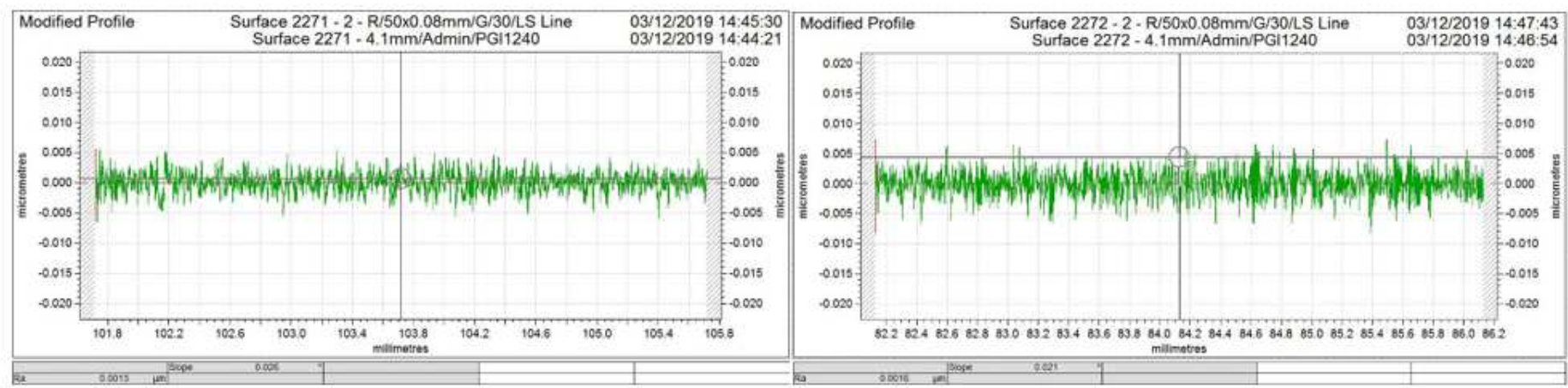

(3)

(c) Result of roughness at $\mathrm{ABCD}$ point

\section{Figure 9}

Measurement results by Taylor Hobson Form Talysurf 